\title{
Mixed Simultaneous Iterative Algorithms for the Extended Multiple-Set Split Equality Common Fixed-Point Problem with Lipschitz Quasi-Pseudocontractive Operators
}

\author{
Meixia Li $\mathbb{D}^{1},{ }^{1}$ Xueling Zhou, ${ }^{2}$ and Haitao Che $\mathbb{D}^{1}$ \\ ${ }^{1}$ School of Mathematics and Information Science, Weifang University, Weifang, Shandong 261061, China \\ ${ }^{2}$ College of Mathematics and Systems Science, Shandong University of Science and Technology, Qingdao, Shandong 266590, China \\ Correspondence should be addressed to Meixia Li; limeixia001@163.com
}

Received 15 November 2018; Accepted 30 January 2019; Published 7 March 2019

Academic Editor: Francesco Soldovieri

Copyright (c) 2019 Meixia Li et al. This is an open access article distributed under the Creative Commons Attribution License, which permits unrestricted use, distribution, and reproduction in any medium, provided the original work is properly cited.

In this paper, we study a kind of extended multiple-set split equality common fixed-point problem with Lipschitz quasipseudocontractive operators, which is an extension of multiple-set split equality common fixed-point problem with quasinonexpansive operator. We propose two mixed simultaneous iterative algorithms, in which the selecting of the stepsize does not need any priori information about the operator norms. Furthermore, we prove that the sequences generated by the mixed simultaneous iterative algorithms converge weakly to the solution of this problem. Some numerical results are shown to illustrate the feasibility and efficiency of the proposed algorithms.

\section{Introduction}

Throughout this paper, we always assume that $H_{1}, H_{2}$, and $H_{3}$ are real Hilbert spaces with inner product $\langle\cdot, \cdot\rangle$ and induced norm $\|\cdot\|$. Let $C$ and $Q$ be nonempty closed and convex subsets of $H_{1}$ and $H_{2}$, respectively. $A: H_{1} \longrightarrow H_{2}$ is a bounded linear operators. Let $I$ denote the identity operator. The split feasibility problem (SFP) originally introduced by Censor and Elfving [1] which is to find

$$
x \in C \text { such that } A x \in Q \text {. }
$$

It can be used in various disciplines such as image restoration, radiation therapy treatment planning $[2,3]$. These applications are in finite-dimensional Hilbert spaces [4-7]. It also can be found in an infinite-dimensional real Hilbert space [8$10]$ and references therein.

In [11], Censor et al. introduce the multiple-set split feasibility problem (MSFP) which finds application in intensitymodulated radiation therapy. It is formulated as finding a point $x$ satisfying the following property:

$$
x \in \bigcap_{i=1}^{p} C_{i} \quad \text { such that } A x \in \bigcap_{j=1}^{r} Q_{j},
$$

where $p, r \geq 1$ are integers and $\left\{C_{i}\right\}_{i=1}^{p}$ and $\left\{Q_{j}\right\}_{j=1}^{r}$ are nonempty closed convex subsets of $H_{1}$ and $H_{2}$, respectively. When $p=r=1$, then MSFP (2) is known as SFP (1). About the corresponding results in finite-dimensional spaces, we can see [12-14] and references therein.

Since every closed convex subset of a Hilbert space is the fixed-point set of its associating projection, problems (1) and (2) are all special cases of the so-called multiple-set split common fixed-point problem (MSCFP) which is to find

$$
x \in \bigcap_{i=1}^{p} \operatorname{Fix}\left(S_{i}\right) \quad \text { such that } A x \in \bigcap_{j=1}^{r} \operatorname{Fix}\left(T_{j}\right),
$$

where $p, r \geq 1$ are integers, $\left\{S_{i}\right\}_{i=1}^{p}: H_{1} \longrightarrow H_{1}$ and $\left\{T_{j}\right\}_{j=1}^{r}$ : $\mathrm{H}_{2} \longrightarrow \mathrm{H}_{2}$ are nonlinear operators. In particular, if $p=r=$ 1 , then MSCFP (3) reduces to the split common fixed-point problem (SCFP) [15-17] which is to find

$$
x \in \operatorname{Fix}(S) \quad \text { such that } A x \in \operatorname{Fix}(T),
$$

where $S: H_{1} \longrightarrow H_{1}$ and $T: H_{2} \longrightarrow H_{2}$ are nonlinear operators. 
Recently, Moudafi [18] introduced the split equality common fixed-point problem (SECFP) which is to find

$$
\begin{aligned}
& x \in \operatorname{Fix}(S), \\
& y \in \operatorname{Fix}(T) \\
& \text { such that } A x=B y,
\end{aligned}
$$

where $A: H_{1} \longrightarrow H_{3}$ and $B: H_{2} \longrightarrow H_{3}$ are two bounded linear operators. It allows asymmetric and partial relations between the variables $x$ and $y$. The interest is to cover many situations, for instance, applications in decomposition methods for PDEs, in game theory, and in intensitymodulated radiation therapy (IMRT). At the same time, Moudafi [18] introduced the following iterative method

$$
\begin{aligned}
& x_{n+1}=S\left(x_{n}-\gamma_{n} A^{*}\left(A x_{n}-B y_{n}\right)\right), \\
& y_{n+1}=T\left(y_{n}+\gamma_{n} B^{*}\left(A x_{n+1}-B y_{n}\right)\right)
\end{aligned}
$$

for firmly quasi-nonexpansive mappings $S$ and $T$. The weak convergence property was proved where $\gamma_{n}$ was dependent in the spectral radiuses of $A^{*} A$ and $B^{*} B$. Furthermore, Che and $\mathrm{Li}$ [19] proposed the following iterative algorithm:

$$
\begin{aligned}
u_{n} & =x_{n}-\gamma_{n} A^{*}\left(A x_{n}-B y_{n}\right), \\
x_{n+1} & =\alpha_{n} x_{n}+\left(1-\alpha_{n}\right) S u_{n}, \\
v_{n} & =y_{n}+\gamma_{n} B^{*}\left(A x_{n}-B y_{n}\right), \\
y_{n+1} & =\alpha_{n} y_{n}+\left(1-\alpha_{n}\right) T v_{n}
\end{aligned}
$$

for quasi-nonexpansive mappings $S$ and $T$ and the weak convergence of scheme (7) can also be established. Chang, Wang, and Qin [20] modified the iterative scheme (7) and provided the following framework:

$$
\begin{aligned}
u_{n}= & x_{n}-\gamma_{n} A^{*}\left(A x_{n}-B y_{n}\right), \\
x_{n+1}= & \alpha_{n} x_{n} \\
& +\left(1-\alpha_{n}\right)[(1-\xi) I+\xi S((1-\eta) I+\eta S)] u_{n}, \\
v_{n}= & y_{n}+\gamma_{n} B^{*}\left(A x_{n}-B y_{n}\right), \\
y_{n+1}= & \alpha_{n} y_{n} \\
& +\left(1-\alpha_{n}\right)[(1-\xi) I+\xi T((1-\eta) I+\eta T)] v_{n}
\end{aligned}
$$

for quasi-pseudocontractive mappings $S$ and $T$ with weak convergence property.

In [21], Zhao et al. study the multiple-set split equality common fixed-point problem (MSECFP) which is to find

$$
\begin{aligned}
& x \in \bigcap_{i=1}^{p} \operatorname{Fix}\left(S_{i}\right), \\
& y \in \bigcap_{j=1}^{r} \operatorname{Fix}\left(T_{j}\right)
\end{aligned}
$$

such that $A x=B y$,

where $p, r \geq 1$ are integers and $\left\{S_{i}\right\}_{i=1}^{p}: H_{1} \longrightarrow H_{1}$ and $\left\{T_{j}\right\}_{j=1}^{r}: H_{2} \longrightarrow H_{2}$ are nonlinear operators. In particular, if $p=r=1$, then MSECFP (9) reduces to the split equality common fixed-point problem (SECFP) (5). They proposed the following iterative algorithm:

$$
\begin{aligned}
u_{n} & =x_{n}-\gamma_{n} A^{*}\left(A x_{n}-B y_{n}\right), \\
x_{n+1} & =\alpha_{n}^{0} x_{n}+\alpha_{n}^{1} S_{1}\left(x_{n}\right)+\cdots+\alpha_{n}^{p} S_{p}\left(x_{n}\right) \\
v_{n} & =y_{n}+\gamma_{n} B^{*}\left(A x_{n}-B y_{n}\right), \\
y_{n+1} & =\beta_{n} v_{n}+\left(1-\beta_{n}\right) T_{j(n)}\left(v_{n}\right)
\end{aligned}
$$

for quasi-nonexpansive mappings, where $\left\{\alpha_{n}^{i}\right\} \subset[0,1], i=$ $0,1,2, \ldots, p ; n=1,2, \ldots$ such that $\sum_{i=0}^{p} \alpha_{n}^{i}=1$ for every $n=1,2, \ldots$ and $\left\{\beta_{n}\right\} \subset[0,1] . j(n)=n(\bmod r)+1$. Weak convergence results were obtained under certain assumption conditions.

Recently, Li et al. [22] studied the extended split equality common fixed-point problem (ESECFP) which is to find

$$
\begin{aligned}
& x \in \operatorname{Fix}(S), \\
& y \in \operatorname{Fix}(T)
\end{aligned}
$$

such that $A x=B y \in K$,

where $\mathrm{KC}_{3}$ is a nonempty set. For L-Lipschitz and quasipseudocontractive mappings $S$ and $T$, as well as a kind of special $K=\left\{x \in H_{3}: h(x) \leq 0\right\}$, they proposed the following iterative algorithm:

$$
\begin{aligned}
& \omega_{n}=P_{K_{n}}\left(\frac{A x_{n}+B y_{n}}{2}\right), \\
& K_{n}=\left\{z \in H_{3}: h\left(\frac{A x_{n}+B y_{n}}{2}\right)\right. \\
&\left.+\left\langle\varsigma_{n}, z-\frac{A x_{n}+B y_{n}}{2}\right\rangle \leq 0\right\}, \\
& u_{n}=x_{n}-\xi_{n} A^{*}\left(A x_{n}-\omega_{n}\right), \\
& x_{n+1}=\alpha_{n} x_{n}+\left(1-\alpha_{n}\right) U u_{n}, \\
& v_{n}=y_{n}-\xi_{n} B^{*}\left(B y_{n}-\omega_{n}\right), \\
& y_{n+1}=\alpha_{n} y_{n}+\left(1-\alpha_{n}\right) V v_{n},
\end{aligned}
$$

where $U:=(1-\xi) I+\xi S((1-\eta) I+\eta S), V:=(1-\xi) I+\xi T((1-\eta) I+$ $\eta T), 0<\xi<\eta<1 /\left(1+\sqrt{1+L^{2}}\right)$, and $\varsigma_{n} \in \partial h\left(\left(A x_{n}+B y_{n}\right) / 2\right)$. They also proved the weak convergence results under mild conditions.

Inspired by the above results, in this paper, we study the following extended multiple-set split equality common fixedpoint problem (EMSECFP) which is to find

$$
\begin{aligned}
& x \in \bigcap_{i=1}^{p} \operatorname{Fix}\left(S_{i}\right), \\
& y \in \bigcap_{j=1}^{r} \operatorname{Fix}\left(T_{j}\right)
\end{aligned}
$$


where $p, r \geq 1$ are integers and $S_{i}: H_{1} \longrightarrow H_{1}(i=1,2, \ldots, p)$ and $T_{j}: H_{2} \longrightarrow H_{2}(j=1,2, \ldots r)$ are $L$-Lipschitzian mappings with $L \geq 1$ and quasi-pseudocontractive mappings with nonempty fixed-point sets which is more general than the class of quasi-nonexpansive mappings. When $\mathrm{K}=\mathrm{H}_{3}$, the EMSECFP (13) is MSECFP (9). When $p=r=1$, the EMSECFP (13) is ESECFP (11). Therefore, EMSECFP (13) is the extension of MSECFP (9) and ESECFP (11). In this paper, we propose two mixed simultaneous iterative algorithms for solving EMSECFP (13), which avoids using the projection and the selecting of stepsize which do not depend on the operator norms. Furthermore, we prove that the sequences generated by the algorithms weakly converge to a solution of the EMSECFP (13). Numerical examples show the feasibility and efficiency of these algorithms.

\section{Preliminaries}

In this paper, we use $x_{n} \rightarrow x$ and $x_{n} \rightarrow x$ to indicate that the sequence $\left\{x_{n}\right\}$ converges weakly to $x$ and $\left\{x_{n}\right\}$ converges strongly to $x$, respectively. We write $\omega_{w}\left(x_{k}\right)=\left\{x: \exists x_{k_{j}} \rightarrow x\right\}$ to stand for the weak $\omega$-limit set of $\left\{x_{k}\right\}$ and use $\Omega$ to stand for the solution set of the EMSECFP (13).

Definition 1. A mapping $T: H \longrightarrow H$ is called

(i) Nonexpansive if $\|T x-T y\| \leq\|x-y\|$ for all $x, y \in H$.

(ii) Quasi-nonexpansive, if $\operatorname{Fix}(T) \neq \emptyset$ and $\left\|T x-x^{*}\right\| \leq$ $\left\|x-x^{*}\right\|$ for all $x \in H$ and $x^{*} \in \operatorname{Fix}(T)$.

(iii) Quasi-pseudocontractive, if $\operatorname{Fix}(T) \neq \emptyset$ and $\| T x-$ $x^{*}\left\|^{2} \leq\right\| x-x^{*}\left\|^{2}+\right\| T x-x \|^{2}$ for all $x \in H$ and $x^{*} \in \operatorname{Fix}(T)$.

Definition 2. An operator $T: H \longrightarrow H$ is called demiclosed at the origin, for any sequence $\left\{x_{n}\right\}$ which weakly converges to $x$, and if the sequence $\left\{T x_{n}\right\}$ strongly converges to 0 , then $T x=0$.

In the proof of our results, we need the following lemmas.

Lemma 3 (see [23]). Let $H$ be a real Hilbert space, then the following conclusions hold:

$$
\begin{gathered}
\|x-y\|^{2}=\|x\|^{2}+\|y\|^{2}-2\langle x, y\rangle, \\
\text { for all } x, y \in H, \\
\|\alpha x+(1-\alpha) y\|^{2}=\alpha\|x\|^{2}+(1-\alpha)\|y\|^{2} \\
-\alpha(1-\alpha)\|x-y\|, \\
\quad \text { for all } x, y \in H, \alpha \in[0,1] .
\end{gathered}
$$

Lemma 4 (see [20]). Let $H$ be a real Hilbert space and $T$ : $H \longrightarrow H$ be a L-Lipschitzian mapping with $L \geq 1$. Denote

$$
U:=(1-\xi) I+\xi T((1-\eta) I+\eta T) .
$$

If $0<\xi<\eta<1 /\left(1+\sqrt{1+L^{2}}\right)$, then the following conclusions hold:

(i) $\operatorname{Fix}(T)=\operatorname{Fix}(T((1-\eta) I+\eta T))=\operatorname{Fix}(U)$.

(ii) If $T$ is demiclosed at 0 , then $U$ is also demiclosed at 0 . (iii) In addition, if $T: H \longrightarrow H$ is quasi-pseudocontractive, then the mapping $U$ is quasi-nonexpansive; that is,

$$
\begin{aligned}
& \left\|U x-u^{*}\right\| \leq\left\|x-u^{*}\right\|, \\
& \forall x \in H, u^{*} \in \operatorname{Fix}(T)=\operatorname{Fix}(U) .
\end{aligned}
$$

Lemma 5 (see [21]). Let $H$ be a real Hilbert space. Then

$$
\begin{aligned}
& \left\|\alpha_{0} x_{0}+\alpha_{1} x_{1}+\cdots+\alpha_{r} x_{r}\right\|^{2} \\
& \quad \leq \sum_{i=0}^{r} \alpha_{i}\left\|x_{i}\right\|^{2}-\alpha_{s} \alpha_{t}\left\|x_{s}-x_{t}\right\|^{2},
\end{aligned}
$$

for any $s, t \in\{0,1,2, \ldots, r\}$ and for $x_{i} \in H, i=0,1,2, \ldots, r$ with $\alpha_{0}+\alpha_{1}+\cdots+\alpha_{r}=1$ and $0<\alpha_{i}<1$.

Lemma 6 (see [16]). Let $H$ be a real Hilbert space and $T$ : $H \rightarrow H$ be a quasi-nonexpansive mapping. Set $T_{\alpha}=\alpha I+(1-$ $\alpha) T$ for $\alpha \in[0,1)$. Then the following properties are reached for all $(x, q) \in H \times F(T)$ :

(i) $\langle x-T x, x-q\rangle \geq(1 / 2)\|x-T x\|^{2}$ and $\langle x-T x, q-T x\rangle \leq$ $(1 / 2)\|x-T x\|^{2}$;

(ii) $\left\|T_{\alpha} x-q\right\|^{2} \leq\|x-q\|^{2}-\alpha(1-\alpha)\|T x-x\|^{2}$;

(iii) $\left\langle x-T_{\alpha} x, x-q\right\rangle \geq((1-\alpha) / 2)\|x-T x\|^{2}$.

\section{Main Results}

In this section, we give the following assumption.

Assumption 7. Assume that

(i) $H_{1}, H_{2}$, and $H_{3}$ are real Hilbert spaces and $R^{n}$ is $n$ dimensional Euclidean space. $A: H_{1} \longrightarrow H_{3}$ and $B: H_{2} \longrightarrow$ $H_{3}$ are two bounded linear operators; $A^{*}$ and $B^{*}$ are their adjoint operators, respectively.

(ii) $K=\left\{x \in H_{3}: h(x) \leq 0\right\}$, where $h$ : $\mathrm{H}_{3} \longrightarrow \mathrm{R}$ is convex function which is subdifferentiable and its subdifferentials are bounded on bounded sets.

(iii) $S_{i}: H_{1} \longrightarrow H_{1}$ and $T_{j}: H_{2} \longrightarrow H_{2}$ are all LLipschitz, quasi-pseudocontractive with $L \geq 1, \operatorname{Fix}\left(S_{i}\right) \neq \emptyset$ and $\operatorname{Fix}\left(T_{j}\right) \neq \emptyset$ and $S_{i}-I$ and $T_{j}-I$ are demiclosed at origin, where $i=1,2, \ldots, p ; j=1,2, \ldots, r$.

(iv) $U_{i}=(1-\xi) I+\xi S_{i}\left((1-\eta) I+\eta S_{i}\right), i=1,2, \ldots, p$, and $V_{j}=(1-\xi) I+\xi T_{j}\left((1-\eta) I+\eta T_{j}\right), j=1,2, \ldots, r$, where $0<\xi<\eta<1 /\left(1+\sqrt{1+L^{2}}\right)$.

Theorem 8. Let Assumption 7 be true and $\delta>0$ be small enough. Assume that $\left\{\alpha_{n}^{i}\right\} \subset[0,1], i=0,1,2, \ldots, p ; n=$ $1,2, \ldots$, such that $\sum_{i=0}^{p} \alpha_{n}^{i}=1$ for every $n=1,2, \ldots$ and $\left\{\beta_{n}\right\} \subset[0,1] . j(n)=n(\bmod r)+1$. Suppose that $x_{0} \in H_{1}$, 
4

Mathematical Problems in Engineering

$y_{0} \in H_{2}$ are arbitrary and $\left\{x_{n}\right\},\left\{y_{n}\right\},\left\{\omega_{n}\right\},\left\{u_{n}\right\}$, and $\left\{v_{n}\right\}$ are generated by the following iteration scheme:

$$
\begin{aligned}
& \omega_{n}=P_{K_{n}}\left(\frac{A x_{n}+B y_{n}}{2}\right), \\
& K_{n}=\left\{z \in H_{3}: h\left(\frac{A x_{n}+B y_{n}}{2}\right)\right. \\
& \left.+\left\langle\varsigma_{n}, z-\frac{A x_{n}+B y_{n}}{2}\right\rangle \leq 0\right\}, \\
& u_{n}=x_{n}-\gamma_{n} A^{*}\left(A x_{n}-\omega_{n}\right) \text {, } \\
& x_{n+1}=\alpha_{n}^{0} u_{n}+\alpha_{n}^{1} U_{1} u_{n}+\cdots+\alpha_{n}^{p} U_{p} u_{n}, \\
& v_{n}=y_{n}-\gamma_{n} B^{*}\left(B y_{n}-\omega_{n}\right) \text {, } \\
& y_{n+1}=\beta_{n} v_{n}+\left(1-\beta_{n}\right) V_{j(n)} v_{n} \text {, }
\end{aligned}
$$

where

$$
\varsigma_{n} \in \partial h\left(\frac{A x_{n}+B y_{n}}{2}\right)
$$

and

$$
\begin{aligned}
\gamma_{n} & \in(\delta,(1-\delta) \\
\cdot & \left.\frac{\left\|A x_{n}-\omega_{n}\right\|^{2}+\left\|B y_{n}-\omega_{n}\right\|^{2}}{\left\|A^{*}\left(A x_{n}-\omega_{n}\right)\right\|^{2}+\left\|B^{*}\left(B y_{n}-\omega_{n}\right)\right\|^{2}}\right) .
\end{aligned}
$$

Similarly,

$$
\begin{aligned}
\left\|v_{n}-y^{*}\right\|^{2}= & \left\|y_{n}-y^{*}\right\|^{2}+\gamma_{n}^{2}\left\|B^{*}\left(B y_{n}-\omega_{n}\right)\right\|^{2} \\
& -\gamma_{n}\left\|B y_{n}-\omega^{*}\right\|^{2}-\gamma_{n}\left\|B y_{n}-\omega_{n}\right\|^{2} \\
& +\gamma_{n}\left\|\omega_{n}-\omega^{*}\right\|^{2} .
\end{aligned}
$$

By (23) and (24), we have

$$
\begin{aligned}
\| u_{n}- & x^{*}\left\|^{2}+\right\| v_{n}-y^{*} \|^{2} \\
= & \left\|x_{n}-x^{*}\right\|^{2}+\left\|y_{n}-y^{*}\right\|^{2}+\gamma_{n}^{2}\left\|A^{*}\left(A x_{n}-\omega_{n}\right)\right\|^{2} \\
& -\gamma_{n}\left\|A x_{n}-\omega^{*}\right\|^{2}-\gamma_{n}\left\|A x_{n}-\omega_{n}\right\|^{2} \\
& +\gamma_{n}^{2}\left\|B^{*}\left(B y_{n}-\omega_{n}\right)\right\|^{2}-\gamma_{n}\left\|B y_{n}-\omega^{*}\right\|^{2} \\
& -\gamma_{n}\left\|B y_{n}-\omega_{n}\right\|^{2}+2 \gamma_{n}\left\|\omega_{n}-\omega^{*}\right\|^{2} .
\end{aligned}
$$

Assume $\Omega \neq \emptyset$, $\liminf _{n \longrightarrow \infty} \alpha_{n}^{0} \alpha_{n}^{i}>0, i=1,2, \ldots, p$, and $\beta_{n} \subset(\sigma, 1-\sigma)$ for small enough $\sigma>0$. Then $\left(x_{n}, y_{n}\right) \rightarrow(\tilde{x}, \tilde{y})$, $\left(u_{n}, v_{n}\right) \rightarrow(\tilde{x}, \tilde{y})$, and $\omega_{n} \rightarrow \widetilde{\omega}:=A \tilde{x}=B \tilde{y}$, where $(\tilde{x}, \tilde{y}) \in \Omega$.

Proof. It is obvious that $K \subset K_{n}$ for any $n \in N$. Let $\left(x^{*}, y^{*}\right) \in$ $\Omega$, that is, $x^{*} \in \bigcap_{i=1}^{p} \operatorname{Fix}\left(S_{i}\right), y^{*} \in \bigcap_{j=1}^{r} \operatorname{Fix}\left(T_{j}\right)$ and $\omega^{*}=$ $A x^{*}=B y^{*} \in K$. By Lemma 3, we have

$$
\begin{aligned}
\left\|\omega_{n}-\omega^{*}\right\|= & \left\|P_{K_{n}}\left(\frac{A x_{n}+B y_{n}}{2}\right)-P_{K_{n}}\left(\omega^{*}\right)\right\|^{2} \\
\leq & \left\|\frac{1}{2}\left(A x_{n}-\omega^{*}\right)+\frac{1}{2}\left(B y_{n}-\omega^{*}\right)\right\|^{2} \\
= & \frac{1}{2}\left\|A x_{n}-\omega^{*}\right\|^{2}+\frac{1}{2}\left\|B y_{n}-\omega^{*}\right\|^{2} \\
& -\frac{1}{4}\left\|A x_{n}-B y_{n}\right\|^{2} .
\end{aligned}
$$

Combining (22) and (25), we have

$$
\begin{aligned}
\| u_{n}- & x^{*}\left\|^{2}+\right\| v_{n}-y^{*} \|^{2} \\
\leq & \left\|x_{n}-x^{*}\right\|^{2}+\left\|y_{n}-y^{*}\right\|^{2}+\gamma_{n}^{2}\left\|A^{*}\left(A x_{n}-\omega_{n}\right)\right\|^{2} \\
& -\gamma_{n}\left\|A x_{n}-\omega_{n}\right\|^{2}+\gamma_{n}^{2}\left\|B^{*}\left(B y_{n}-\omega_{n}\right)\right\|^{2} \\
& -\gamma_{n}\left\|B y_{n}-\omega_{n}\right\|^{2}-\frac{1}{2} \gamma_{n}\left\|A x_{n}-B y_{n}\right\|^{2} .
\end{aligned}
$$
Lemma $4, U_{i}, i=1,2, \ldots, p$, are quasi-nonexpansive. Furthermore, from Lemma 5, we have

$$
\begin{aligned}
\left\|x_{n+1}-x^{*}\right\|^{2} \leq & \alpha_{n}^{0}\left\|u_{n}-x^{*}\right\|^{2}+\alpha_{n}^{1}\left\|U_{1}\left(u_{n}\right)-x^{*}\right\|^{2} \\
& +\cdots+\alpha_{n}^{p}\left\|U_{p}\left(u_{n}\right)-x^{*}\right\|^{2} \\
& -\alpha_{n}^{0} \alpha_{n}^{1}\left\|U_{1}\left(u_{n}\right)-u_{n}\right\|^{2} \\
\leq & \alpha_{n}^{0}\left\|u_{n}-x^{*}\right\|^{2}+\alpha_{n}^{1}\left\|u_{n}-x^{*}\right\|^{2}+\cdots \\
& +\alpha_{n}^{p}\left\|u_{n}-x^{*}\right\|^{2} \\
& -\alpha_{n}^{0} \alpha_{n}^{1}\left\|U_{1}\left(u_{n}\right)-u_{n}\right\|^{2} \\
= & \left\|u_{n}-x^{*}\right\|^{2}-\alpha_{n}^{0} \alpha_{n}^{1}\left\|U_{1}\left(u_{n}\right)-u_{n}\right\|^{2} .
\end{aligned}
$$

Since $S_{i}, i=1,2, \ldots, p$, are quasi-pseudocontractive, from 
Similarly, since $T_{j}, j=1,2, \ldots, r$ are quasi-pseudocontractive, from Lemma $4, V_{j}, j=1,2, \ldots, r$, are quasinonexpansive. By Lemma 6 (ii), we have

$$
\begin{aligned}
\left\|y_{n+1}-y^{*}\right\|^{2} \leq & \left\|v_{n}-y^{*}\right\|^{2} \\
& -\beta_{n}\left(1-\beta_{n}\right)\left\|V_{j(n)}\left(v_{n}\right)-v_{n}\right\|^{2} .
\end{aligned}
$$

Combining (27) and (28) with (26), we have

$$
\begin{aligned}
\left\|x_{n+1}-x^{*}\right\|^{2}+\left\|y_{n+1}-y^{*}\right\|^{2} \\
\leq\left\|u_{n}-x^{*}\right\|^{2}+\left\|v_{n}-y^{*}\right\|^{2}-\alpha_{n}^{0} \alpha_{n}^{1}\left\|U_{1}\left(u_{n}\right)-u_{n}\right\|^{2} \\
\quad-\beta_{n}\left(1-\beta_{n}\right)\left\|v_{n}-V_{j(n)}\left(v_{n}\right)\right\|^{2} \\
\leq\left\|x_{n}-x^{*}\right\|^{2}+\left\|y_{n}-y^{*}\right\|^{2}+\gamma_{n}^{2}\left\|A^{*}\left(A x_{n}-\omega_{n}\right)\right\|^{2} \\
\quad+\gamma_{n}^{2}\left\|B^{*}\left(B y_{n}-\omega_{n}\right)\right\|^{2}-\gamma_{n}\left\|A x_{n}-\omega_{n}\right\|^{2} \\
\quad-\gamma_{n}\left\|B y_{n}-\omega_{n}\right\|^{2}-\frac{1}{2} \gamma_{n}\left\|A x_{n}-B y_{n}\right\|^{2} \\
\quad-\alpha_{n}^{0} \alpha_{n}^{1}\left\|U_{1}\left(u_{n}\right)-u_{n}\right\|^{2} \\
\quad-\beta_{n}\left(1-\beta_{n}\right)\left\|V_{j(n)}\left(v_{n}\right)-v_{n}\right\|^{2} .
\end{aligned}
$$

Since $\gamma_{n} \in\left(\delta,(1-\delta)\left(\left(\left\|A x_{n}-\omega_{n}\right\|^{2}+\left\|B y_{n}-\omega_{n}\right\|^{2}\right) /\left(\| A^{*}\left(A x_{n}-\right.\right.\right.\right.$ $\left.\left.\left.\left.\omega_{n}\right)\left\|^{2}+\right\| B^{*}\left(B y_{n}-\omega_{n}\right) \|^{2}\right)\right)\right)$, one has

$$
\begin{aligned}
\gamma_{n}^{2} & \left(\left\|A^{*}\left(A x_{n}-\omega_{n}\right)\right\|^{2}+\left\|B^{*}\left(B y_{n}-\omega_{n}\right)\right\|^{2}\right) \\
& -\gamma_{n}\left(\left\|A x_{n}-\omega_{n}\right\|^{2}+\left\|B y_{n}-\omega_{n}\right\|^{2}\right) \\
& =\gamma_{n}\left[\gamma_{n}\left(\left\|A^{*}\left(A x_{n}-\omega_{n}\right)\right\|^{2}+\left\|B^{*}\left(B y_{n}-\omega_{n}\right)\right\|^{2}\right)\right. \\
& \left.-\left(\left\|A x_{n}-\omega_{n}\right\|^{2}+\left\|B y_{n}-\omega_{n}\right\|^{2}\right)\right] \\
& \leq-\delta \gamma_{n}\left(\left\|A x_{n}-\omega_{n}\right\|^{2}+\left\|B y_{n}-\omega_{n}\right\|^{2}\right) \leq 0 .
\end{aligned}
$$

As a result, we have from (29) and (30) that

$$
\begin{gathered}
\left\|x_{n+1}-x^{*}\right\|^{2}+\left\|y_{n+1}-y^{*}\right\|^{2} \\
\leq\left\|x_{n}-x^{*}\right\|^{2}+\left\|y_{n}-y^{*}\right\|^{2} .
\end{gathered}
$$

We see that the sequence $\left\{\left\|x_{n}-x^{*}\right\|^{2}+\left\|y_{n}-y^{*}\right\|^{2}\right\}$ is decreasing and lower bounded by 0 . Consequently it converges to some finite limit. Hence the sequences $\left\{x_{n}\right\}$ and $\left\{y_{n}\right\}$ are bounded.

According to the assumption of $\left\{\gamma_{n}\right\}$ and (29), we have

$$
\lim _{n \longrightarrow \infty}\left\|A x_{n}-B y_{n}\right\|=0 \text {. }
$$

Furthermore, by the conditions on $\left\{\alpha_{n}^{i}\right\}(0 \leq i \leq p)$ and $\left\{\beta_{n}\right\}$, we obtain

$$
\lim _{n \longrightarrow \infty}\left\|U_{1}\left(u_{n}\right)-u_{n}\right\|=\lim _{n \longrightarrow \infty}\left\|V_{j(n)}\left(v_{n}\right)-v_{n}\right\|=0 .
$$

From Lemma 5, (19) and the conference of (27) for $2 \leq$ $i \leq p$, we have

$$
\lim _{n \longrightarrow \infty}\left\|U_{i}\left(u_{n}\right)-u_{n}\right\|=0
$$

and

$$
\begin{aligned}
& \lim _{n \longrightarrow \infty}\left\|A x_{n}-\omega_{n}\right\|=0, \\
& \lim _{n \longrightarrow \infty}\left\|B y_{n}-\omega_{n}\right\|=0,
\end{aligned}
$$

which combining (19) implies that

$$
\lim _{n \longrightarrow \infty}\left\|u_{n}-x_{n}\right\|=\lim _{n \longrightarrow \infty}\left\|\gamma_{n} A^{*}\left(A x_{n}-\omega_{n}\right)\right\|=0
$$

and

$$
\lim _{n \longrightarrow \infty}\left\|v_{n}-y_{n}\right\|=\lim _{n \longrightarrow \infty}\left\|\gamma_{n} B^{*}\left(B y_{n}-\omega_{n}\right)\right\|=0 .
$$

From (33), (34), and (36), we can conclude that

$$
\lim _{n \rightarrow \infty}\left\|U_{i}\left(u_{n}\right)-x_{n}\right\|=0, \quad \text { for } 1 \leq i \leq p .
$$

According to (36), (38), and

$$
\begin{gathered}
\left\|x_{n+1}-x_{n}\right\| \leq \alpha_{n}^{0}\left\|u_{n}-x_{n}\right\|+\alpha_{n}^{1}\left\|U_{1}\left(u_{n}\right)-x_{n}\right\|+\cdots \\
+\alpha_{n}^{p}\left\|U_{p}\left(u_{n}\right)-x_{n}\right\|
\end{gathered}
$$

we have

$$
\lim _{n \longrightarrow \infty}\left\|x_{n+1}-x_{n}\right\|=0
$$

which infers that $\left\{x_{n}\right\}$ is asymptotically regular. From (33) and (37), we have

$$
\lim _{n \rightarrow \infty}\left\|V_{j(n)}\left(v_{n}\right)-y_{n}\right\|=0 .
$$

As a result,

$$
\begin{aligned}
& \lim _{n \longrightarrow \infty}\left\|y_{n+1}-y_{n}\right\| \\
& \quad=\lim _{n \longrightarrow \infty}\left\|\beta_{n}\left(v_{n}-y_{n}\right)+\left(1-\beta_{n}\right)\left(V_{j(n)}\left(v_{n}\right)-y_{n}\right)\right\| \\
& \quad=0,
\end{aligned}
$$

which implies that $\left\{y_{n}\right\}$ is asymptotically regular.

It follows from

$$
\left\|v_{n+1}-v_{n}\right\| \leq\left\|v_{n+1}-y_{n+1}\right\|+\left\|y_{n+1}-y_{n}\right\|+\left\|y_{n}-v_{n}\right\|
$$

that

$$
\lim _{n \longrightarrow \infty}\left\|v_{n+1}-v_{n}\right\|=0
$$

Furthermore,

$$
\lim _{n \rightarrow \infty}\left\|v_{n+j}-v_{n}\right\|=0, \quad 1 \leq j \leq r .
$$

Taking $(\tilde{x}, \tilde{y}) \in w_{\omega}\left(x_{n}, y_{n}\right)$, from $\lim _{n \longrightarrow \infty}\left\|u_{n}-x_{n}\right\|=0$ and $\lim _{n \rightarrow \infty}\left\|v_{n}-y_{n}\right\|=0$, we have $(\tilde{x}, \tilde{y}) \in w_{\omega}\left(u_{n}, v_{n}\right)$. Let an 
index $j \in\{1,2, \ldots, r\}$ be fixed. Noting that the pool of indexes is finite, from (45) and the boundedness of $\left\{v_{n}\right\}$, there exists a subsequence $\left\{v_{n_{m}}\right\} \subset\left\{v_{n}\right\}$ such that $v_{n_{m}} \rightarrow \tilde{y}$ as $n \longrightarrow \infty$ and $j\left(n_{m}\right)=j$ for all $m$. It is easy to see from (33) that

$$
\begin{aligned}
& \lim _{m \longrightarrow \infty}\left\|V_{j}\left(v_{n_{m}}\right)-v_{n_{m}}\right\|=\lim _{m \longrightarrow \infty}\left\|V_{j\left(n_{m}\right)}\left(v_{n_{m}}\right)-v_{n_{m}}\right\| \\
& \quad=0 .
\end{aligned}
$$

According to the demiclosednesses of $U_{i}-I$ and $V_{j}-I$ at 0 , it yields from (33), (34) and (46) that $U_{i}(\tilde{x})=\tilde{x}$ and $V_{j}(\tilde{y})=\tilde{y}$ for $1 \leq i \leq p$ and $1 \leq j \leq r$. Hence, $\tilde{x} \in \bigcap_{i=1}^{p} F\left(U_{i}\right)$ and $\tilde{y} \in \bigcap_{j=1}^{r} F\left(V_{j}\right)$. From Lemma 4 , we have $\tilde{x} \in \bigcap_{i=1}^{p} F\left(S_{i}\right)$ and $\tilde{y} \in \bigcap_{j=1}^{r} F\left(T_{j}\right)$.

Furthermore, $A \tilde{x}-B \tilde{y} \in w_{\omega}\left(A x_{n}-B y_{n}\right)$ and the weakly lower semicontinuity of the norm imply that

$$
\|A \tilde{x}-B \tilde{y}\| \leq \liminf _{n \longrightarrow \infty}\left\|A x_{n}-B y_{n}\right\|=0,
$$

that is, $A \tilde{x}=B \tilde{y}$.

From $\lim _{n \longrightarrow \infty}\left\|A x_{n}-\omega_{n}\right\|=0$ and $\lim _{n \longrightarrow \infty}\left\|B y_{n}-\omega_{n}\right\|=0$, we have

$$
\omega_{n} \rightarrow \tilde{\omega}=A \tilde{x}=B \tilde{y} .
$$

As follows, we prove $\widetilde{\omega} \in K$.

According to the boundedness of $\left\{s_{n}\right\}$, there exists a constant $\bar{M}>0$, such that $\left\|\varsigma_{n}\right\| \leq \bar{M}$.

Noting that $\omega_{n}=P_{K_{n}}\left(\left(A x_{n}+B y_{n}\right) / 2\right) \in K_{n}$, we obtain

$$
h\left(\frac{A x_{n}+B y_{n}}{2}\right)+\left\langle\varsigma_{n}, \omega_{n}-\frac{A x_{n}+B y_{n}}{2}\right\rangle \leq 0 \text {. }
$$

As a result,

$$
\begin{aligned}
h\left(\frac{A x_{n}+B y_{n}}{2}\right) & \leq-\left\langle\varsigma_{n}, \omega_{n}-\frac{A x_{n}+B y_{n}}{2}\right\rangle \\
& \leq \bar{M}\left\|\omega_{n}-\frac{A x_{n}+B y_{n}}{2}\right\| .
\end{aligned}
$$

From the lower semicontinuity of $h,(49)$, and (50), we have

$$
\begin{aligned}
h(\widetilde{\omega}) & \leq \liminf _{n \longrightarrow \infty} h\left(\frac{A x_{n}+B y_{n}}{2}\right) \\
& \leq \bar{M} \liminf _{n \longrightarrow \infty}\left\|\omega_{n}-\frac{A x_{n}+B y_{n}}{2}\right\|=0 .
\end{aligned}
$$

Thus $\widetilde{\omega} \in K$.

Next, we prove the uniqueness of the weak cluster point of $\left\{\left(x_{n}, y_{n}\right)\right\}$. Assume that $(\bar{x}, \bar{y})$ be other weak cluster point of $\left\{\left(x_{n}, y_{n}\right)\right\}$, then $(\bar{x}, \bar{y}) \in \Gamma$. Define $\rho_{n}(x, y)=\left\|x_{n}-x\right\|^{2}+$ $\left\|y_{n}-y\right\|^{2}$, then

$$
\begin{aligned}
\rho_{n}(\tilde{x}, \tilde{y})= & \left\|x_{n}-\bar{x}\right\|^{2}+\|\bar{x}-\tilde{x}\|^{2}+2\left\langle x_{n}-\bar{x}, \bar{x}-\tilde{x}\right\rangle \\
& +\left\|y_{n}-\bar{y}\right\|^{2}+\|\bar{y}-\tilde{y}\|^{2} \\
& +2\left\langle y_{n}-\bar{y}, \bar{y}-\tilde{y}\right\rangle \\
= & \rho_{n}(\bar{x}, \bar{y})+\|\bar{x}-\tilde{x}\|^{2}+2\left\langle x_{n}-\bar{x}, \bar{x}-\tilde{x}\right\rangle \\
& +\|\bar{y}-\tilde{y}\|^{2}+2\left\langle y_{n}-\bar{y}, \bar{y}-\tilde{y}\right\rangle .
\end{aligned}
$$

Without loss of generality, we suppose that $x_{n} \rightarrow \bar{x}, y_{n} \rightarrow \bar{y}$. By passing to the limit in relation (52), we have

$$
\rho(\tilde{x}, \tilde{y})=\rho(\bar{x}, \bar{y})+\|\bar{x}-\tilde{x}\|^{2}+\|\bar{y}-\tilde{y}\|^{2} .
$$

Reserving the role of $(\tilde{x}, \tilde{y})$ and $(\bar{x}, \bar{y})$, we also have

$$
\rho(\bar{x}, \bar{y})=\rho(\tilde{x}, \tilde{y})+\|\tilde{x}-\bar{x}\|^{2}+\|\tilde{y}-\bar{y}\|^{2} .
$$

By adding the two last equalities, we obtain $\tilde{x}=\bar{x}$ and $\tilde{y}=\bar{y}$, which shows that whole sequence $\left\{\left(x_{n}, y_{n}\right)\right\}$ weakly converges to the unique solution of (13). The proof is completed.

Similar to the proof of Theorem 8, we can easily prove the following theorem.

Theorem 9. Let Assumption 7 be true and $\delta>0$ be small enough. Assume that $\left\{\alpha_{n}\right\} \subset[0,1] . i(n)=n(\bmod p)+1$ and $\left\{\beta_{n}^{i}\right\} \subset[0,1], i=0,1,2, \ldots, r ; n=1,2, \ldots$ such that $\sum_{i=0}^{r} \beta_{n}^{i}=$ 1 for every $n=1,2, \ldots$. Suppose that $x_{0} \in H_{1}, y_{0} \in H_{2}$ are arbitrary and $\left\{x_{n}\right\},\left\{y_{n}\right\},\left\{\omega_{n}\right\},\left\{u_{n}\right\}$, and $\left\{v_{n}\right\}$ are generated by the following iteration scheme:

$$
\begin{aligned}
& \omega_{n}=P_{K_{n}}\left(\frac{A x_{n}+B y_{n}}{2}\right), \\
& K_{n}=\left\{z \in H_{3}: h\left(\frac{A x_{n}+B y_{n}}{2}\right)\right. \\
& \left.\quad+\left\langle\varsigma_{n}, z-\frac{A x_{n}+B y_{n}}{2}\right\rangle \leq 0\right\}, \\
& u_{n}=x_{n}-\gamma_{n} A^{*}\left(A x_{n}-\omega_{n}\right), \\
& x_{n+1}=\alpha_{n} u_{n}+\left(1-\alpha_{n}\right) U_{i(n)} u_{n}, \\
& v_{n}=y_{n}-\gamma_{n} B^{*}\left(B y_{n}-\omega_{n}\right), \\
& y_{n+1}=\beta_{n}^{0} v_{n}+\beta_{n}^{1} V_{1} v_{n}+\cdots+\beta_{n}^{r} V_{r} v_{n},
\end{aligned}
$$

where

$$
\varsigma_{n} \in \partial h\left(\frac{A x_{n}+B y_{n}}{2}\right)
$$

and

$$
\begin{aligned}
\gamma_{n} & \in(\delta,(1-\delta) \\
& \left.\cdot \frac{\left\|A x_{n}-\omega_{n}\right\|^{2}+\left\|B y_{n}-\omega_{n}\right\|^{2}}{\left\|A^{*}\left(A x_{n}-\omega_{n}\right)\right\|^{2}+\left\|B^{*}\left(B y_{n}-\omega_{n}\right)\right\|^{2}}\right) .
\end{aligned}
$$

Assume $\Omega \neq \emptyset, \alpha_{n} \subset(\sigma, 1-\sigma)$ for small enough $\sigma>0$ and $\liminf _{n \rightarrow \infty} \beta_{n}^{0} \beta_{n}^{i}>0, i=1,2, \ldots, r$. Then $\left(x_{n}, y_{n}\right) \rightarrow(\tilde{x}, \tilde{y})$, $\left(u_{n}, v_{n}\right) \rightarrow(\tilde{x}, \tilde{y})$, and $\omega_{n} \rightarrow \widetilde{\omega}:=A \tilde{x}=B \tilde{y}$, where $(\tilde{x}, \tilde{y}) \in \Omega$.

\section{Numerical Examples}

In this section, we give an example to show some insight into the behavior of the algorithms presented in this paper. The algorithms presented in Theorems 8 and 9 are denoted as 
TABle 1: Number of iterations and the cpu time for different $\epsilon$ in Algorithm 1.

\begin{tabular}{lcccc}
\hline$\epsilon$ & Iter. & Sec. & \multicolumn{2}{c}{ Fina. } \\
\hline $10^{-2}$ & 12 & 0.002 & $x=(0.0028,0.0014)^{T}$, & $y=(0.0114,-0.0064,-0.0001)^{T}$ \\
$10^{-4}$ & 55 & 0.004 & $x=(-0.2230,0.2539)^{T} \times 10^{-4}$, & $y=(-0.0189,-0.1302,0.1469)^{T} \times 10^{-3}$ \\
$10^{-6}$ & 104 & 0.006 & $x=(-0.1723,0.4902)^{T} \times 10^{-6}$, & $y=(-0.0264,-0.1526,0.1556)^{T} \times 10^{-5}$ \\
\hline
\end{tabular}

Table 2: Number of iterations and the cpu time for different $\epsilon$ in Algorithm 2.

\begin{tabular}{lcccc}
\hline$\epsilon$ & Iter. & Sec. & \multicolumn{2}{c}{ Fina. } \\
\hline $10^{-2}$ & 12 & 0.002 & $x=(0.0044,0.0008)^{T}$, & $y=(0.0106,-0.0080,0.0011)^{T}$ \\
$10^{-4}$ & 50 & 0.005 & $x=(-0.1526,0.5347)^{T} \times 10^{-4}$, & $y=(-0.0096,-0.1518,0.1633)^{T} \times 10^{-3}$ \\
$10^{-6}$ & 112 & 0.014 & $x=(-0.0627,0.4938)^{T} \times 10^{-6}$, & $y=(-0.0229,-0.1262,0.1157)^{T} \times 10^{-5}$ \\
\hline
\end{tabular}

Algorithms 1 and 2 separately. The whole codes are written in Matlab 7.0. All the numerical results are carried out on a personal Lenovo Thinkpad computer with Intel(R) Core(TM) i7-6500U CPU 2.50GHz and RAM 8.00GB.

In this section, we need the following lemmas.

Lemma 10 (see $[24,25])$. For every $k \geq 0$, let $x^{k} \in R^{n}, C_{k}=$ $\left\{x \in R^{n} \mid c\left(x^{k}\right)+\left\langle\xi^{k}, x-x^{k}\right\rangle \leq 0\right\}$. Then for any $x \in R^{n}$, we have

$$
\begin{aligned}
& P_{C_{k}}(x) \\
& = \begin{cases}x-\frac{c\left(x^{k}\right)+\left\langle\xi^{k}, x-x^{k}\right\rangle}{\left\|\xi^{k}\right\|^{2}} \xi^{k}, & \text { if } c\left(x^{k}\right)+\left\langle\xi^{k}, x-x^{k}\right\rangle>0 \\
x, & \text { otherwise. }\end{cases}
\end{aligned}
$$

Lemma 11 (see [26]). Suppose $h: R^{n} \longrightarrow R$ is a finite convex function, then it is subdifferentiable everywhere and its subdifferentials are uniformly bounded on any bounded subset of $R^{n}$.

Example 12. Let $H_{1}=R^{2}, H_{2}=R^{3}$, and $H_{3}=R^{4}$. Assume that $S_{1} x=-3 x, x \in H_{1}, S_{2} x=-4 x, x \in H_{1}, T_{1} x=-6 x, x \in$ $H_{2}, T_{2} x=-7 x, x \in H_{2}, K=\left\{x \in H_{3} \mid h(x)=x_{1}^{2}+2 x_{2}^{2}+4 x_{3}^{2}+\right.$ $\left.x_{4}^{2}-2 \leq 0\right\}$, and

$$
\begin{aligned}
A & =\left(\begin{array}{ll}
0.5836 & 0.9962 \\
0.5118 & 0.3545 \\
0.0826 & 0.9713 \\
0.7196 & 0.3464
\end{array}\right), \\
B & =\left(\begin{array}{lll}
0.8865 & 0.1257 & 0.6938 \\
0.4547 & 0.3089 & 0.0098 \\
0.4134 & 0.7261 & 0.8432 \\
0.2177 & 0.7829 & 0.9223
\end{array}\right) .
\end{aligned}
$$
$B y \in K$.

Find $x \in \bigcap_{i=1}^{2} \operatorname{Fix}\left(S_{i}\right), y \in \bigcap_{j=1}^{2} \operatorname{Fix}\left(T_{j}\right)$ such that $A x=$

It is easy to see that $S_{i}: H_{1} \longrightarrow H_{1}, i=1,2$ and $T_{j}:$ $H_{2} \longrightarrow H_{2}, j=1,2$ are all Lipschitz quasi-pseudocontractive operators, but not quasi-nonexpansive operators. Therefore, the Example 12 cannot be calculated by the algorithms in [21].

In the experiments, we take $\delta=0.6, L=7, \xi=1 / 3 L$, $\eta=1 / 2 L, \alpha_{n}^{0}=\alpha_{n}^{1}=0.25, \alpha_{n}^{2}=0.5$, and $\beta_{n}=0.3$. And the stopping criterion is $\left\|A x_{n}-w_{n}\right\|+\left\|B y_{n}-w_{n}\right\| \leq \epsilon$.

Tables 1 and 2 show the number of iterations, the cpu time, and the final iteration points for different $\epsilon$ when the initial point is $x_{0}=(0.1,0.1)^{T}, y_{0}=(0.1,0.1,0.1)^{T}$ in Algorithms 1 and 2. We denote by Iter., Sec. and Fina. the number of iterations, the cpu time in seconds, and the final iteration points, respectively.

Figures 1 and 2 present the behaviors of $\left\|A x_{n}-B y_{n}\right\|$ for $\epsilon=$ $10^{-4}$ and $\epsilon=10^{-6}$ when the initial point is $x_{0}=(0.1,0.1)^{T}$, $y_{0}=(0.1,0.1,0.1)^{T}$ in Algorithms 1 and 2 .

It is easy to see that the presentation reveals that $A x=B y$.

Furthermore, for testing the stationary property of iterative numbers, we carry out 500 experiments for different initial points which are presented randomly, such as

$$
\begin{aligned}
& x=0.1 * \operatorname{rand}(2,1), \\
& y=0.1 * \operatorname{rand}(3,1) .
\end{aligned}
$$

Figures 3 and 4 illustrate the behaviors of iterative numbers for $\epsilon=10^{-4}$ and $\epsilon=10^{-6}$, which reveals the stationary property of iterative numbers of the Algorithms 1 and 2 .

Especially, Figure 5 illustrates the behaviors of $\left\|x_{n+1}-x_{n}\right\|$ and $\left\|y_{n+1}-y_{n}\right\|$ for $\epsilon=10^{-4}$ in Algorithm 1. Figure 6 shows the behaviors of iterative numbers for different $\epsilon$ in Algorithm 1 .

\section{Data Availability}

The data used to support the findings of this study are included within the article.

\section{Conflicts of Interest}

The authors declare that there are no conflicts of interest regarding the publication of this paper. 

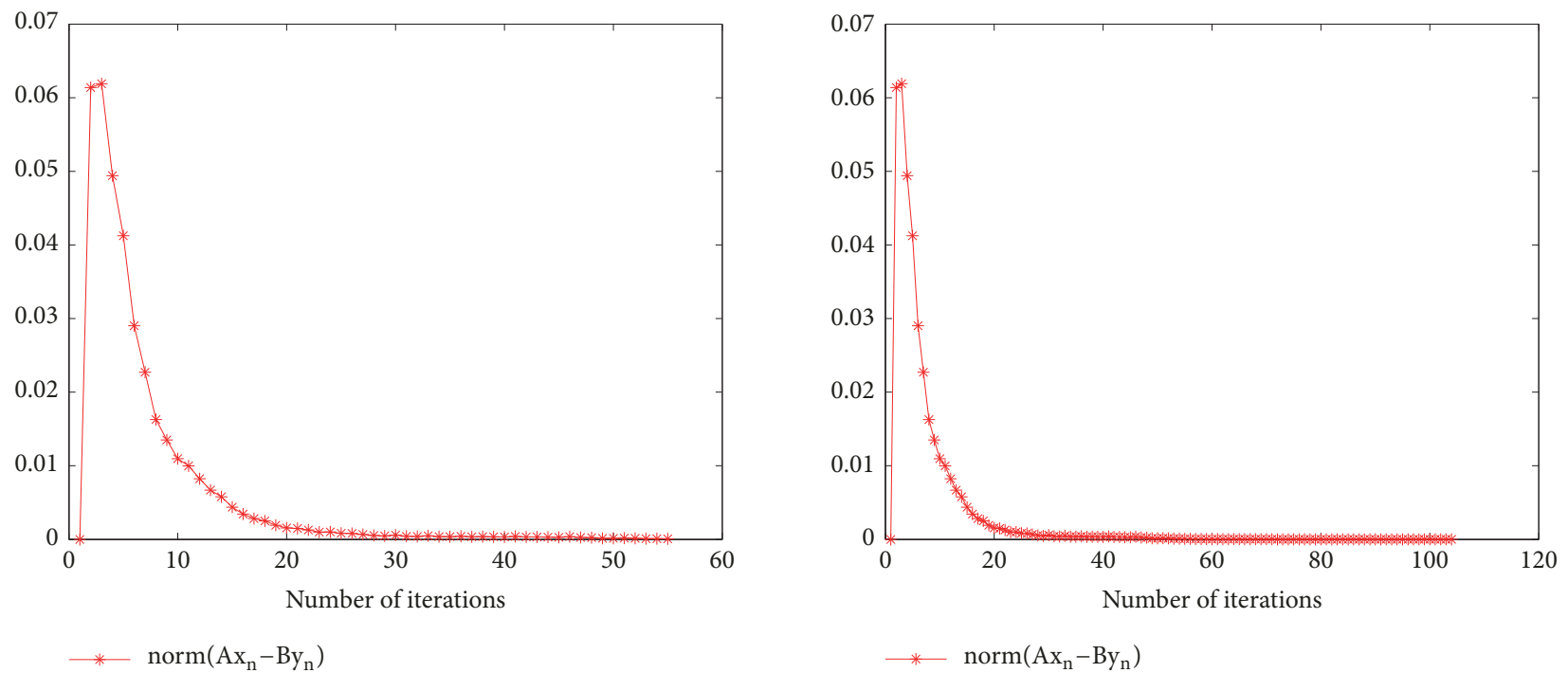

FIGURE 1: The behaviors of $\left\|A x_{n}-B y_{n}\right\|$ for different $\epsilon$ in Algorithm 1.
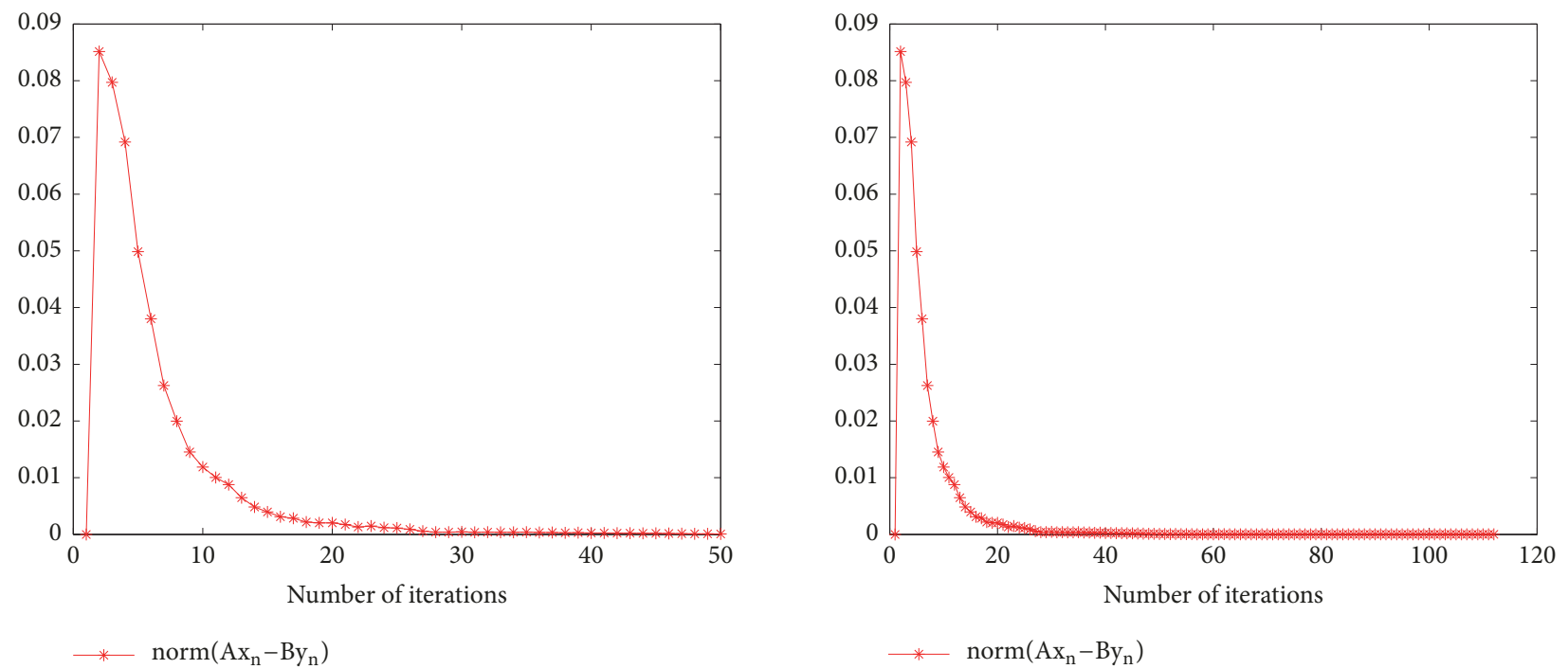

FIgURE 2: The behaviors of $\left\|A x_{n}-B y_{n}\right\|$ for different $\epsilon$ in Algorithm 2.
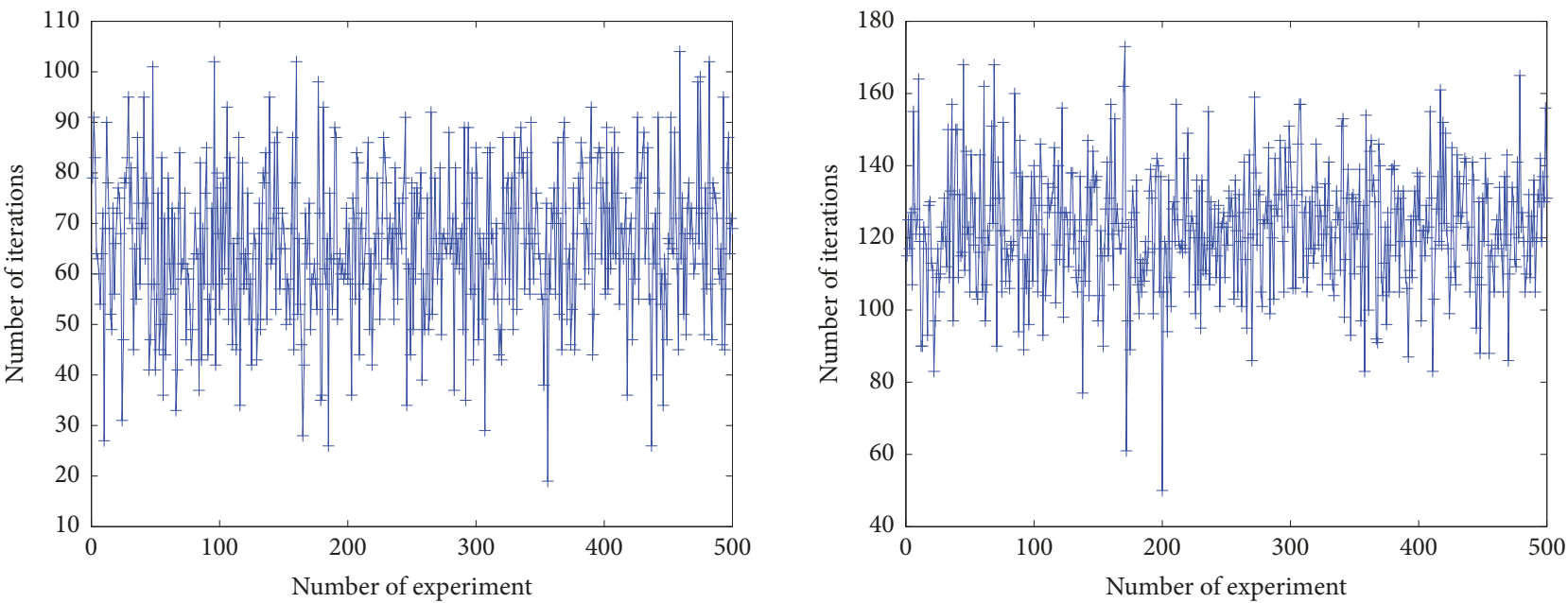

FIGURE 3: The behaviors of iterative numbers for different $\epsilon$ in Algorithm 1. 

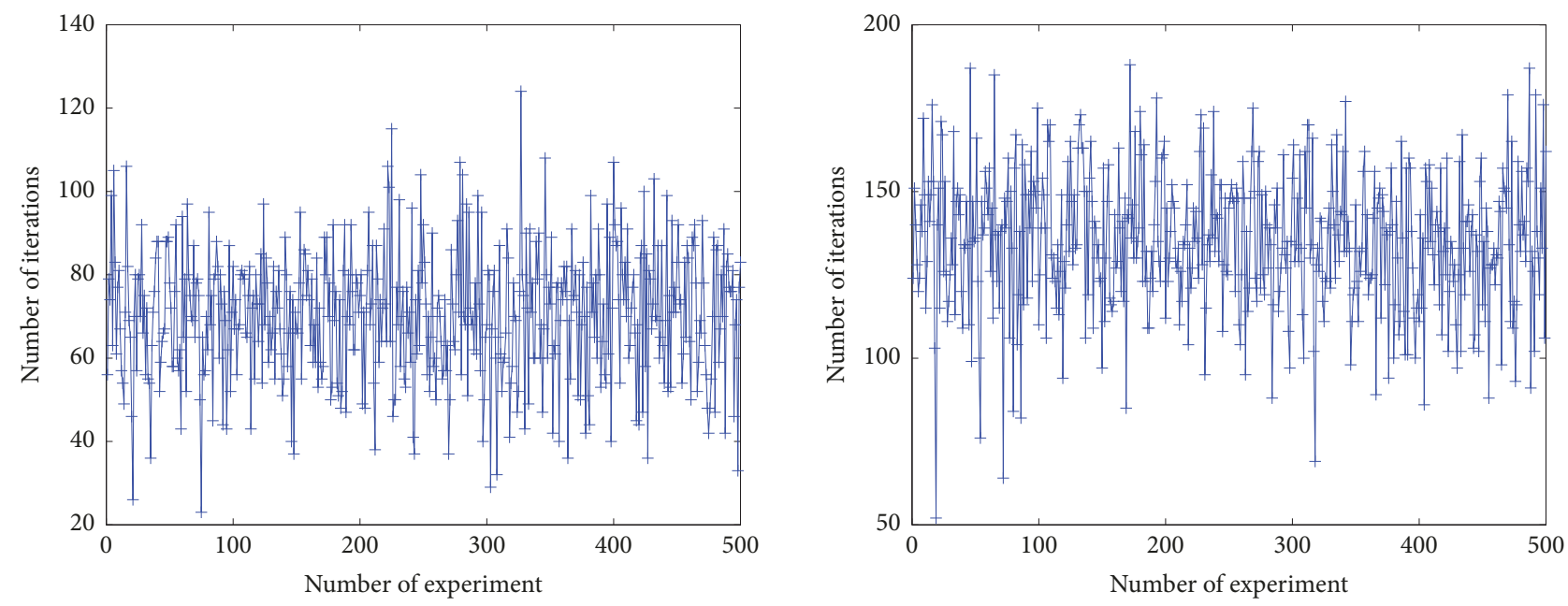

Figure 4: The behaviors of iterative numbers for different $\epsilon$ in Algorithm 2.
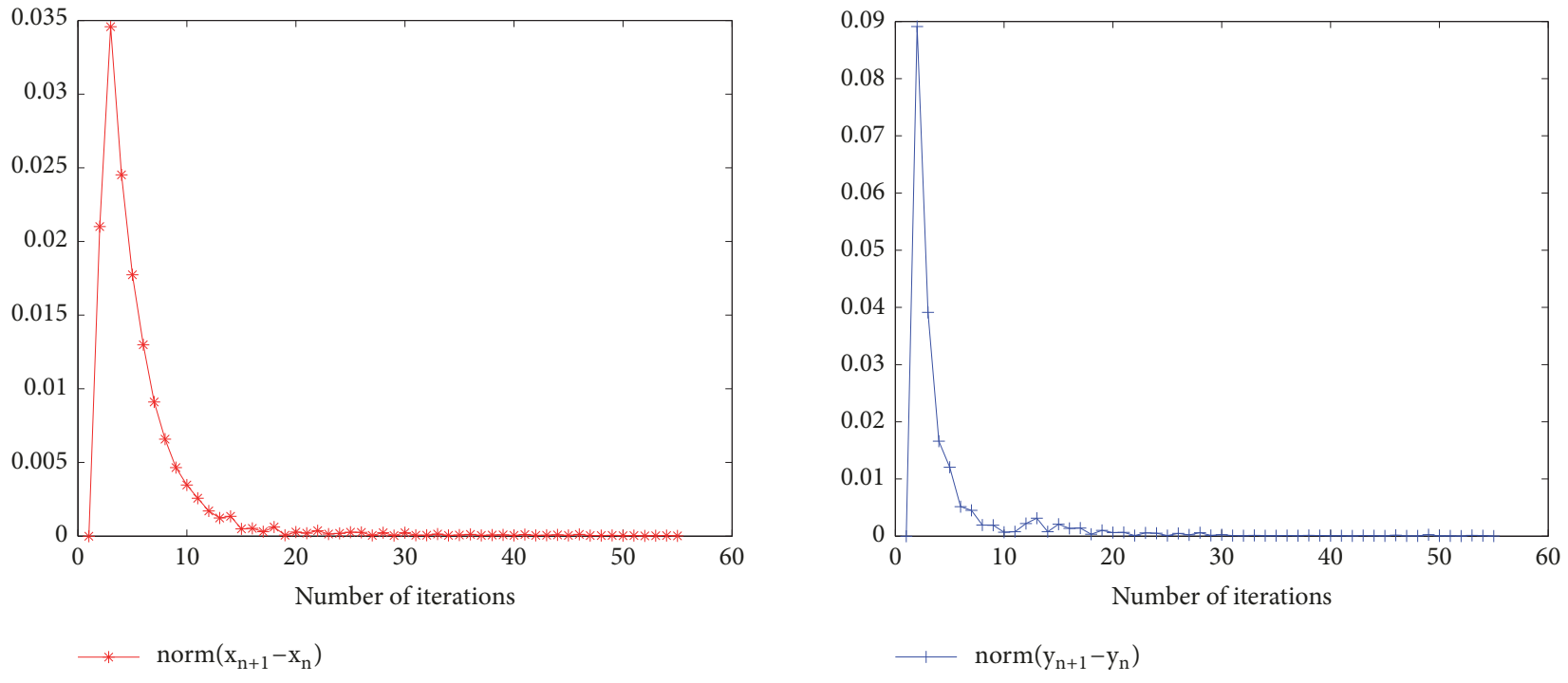

FIGURE 5: The behaviors of $\left\|x_{n+1}-x_{n}\right\|$ and $\left\|y_{n+1}-y_{n}\right\|$ in Algorithm 1 .
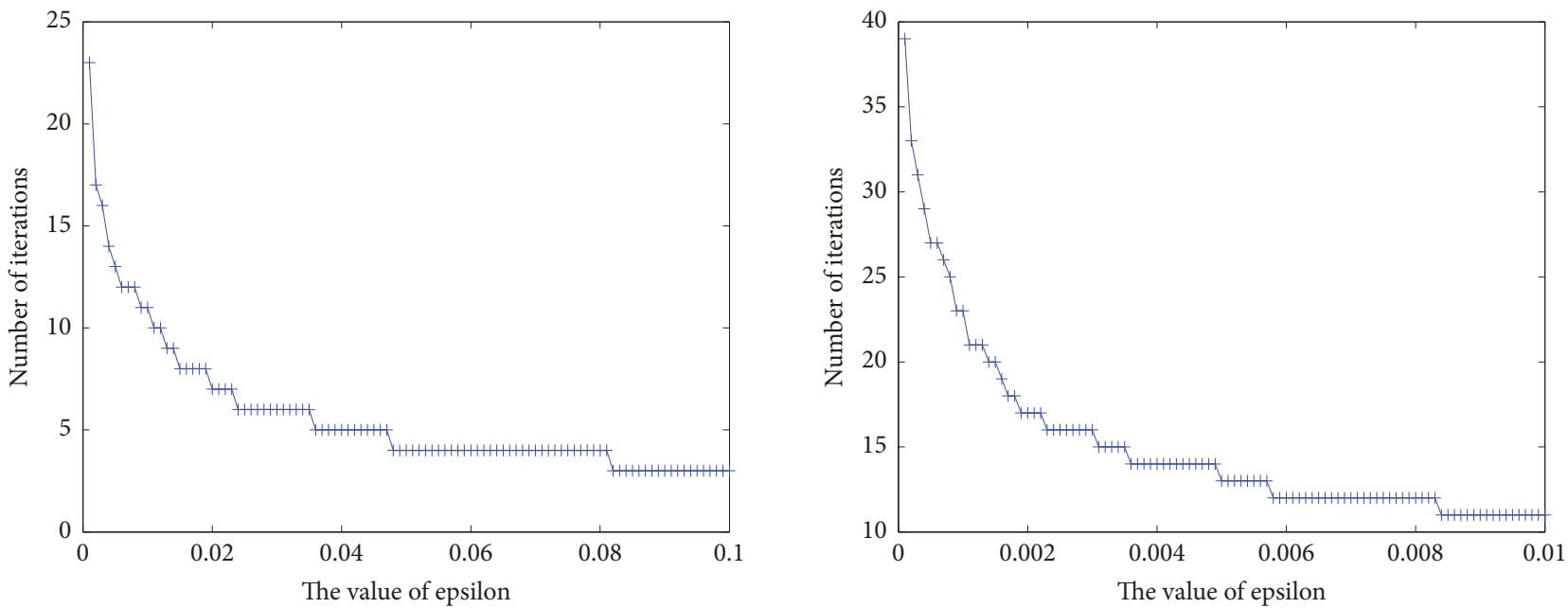

FIGURE 6: The behaviors of iterative numbers for different $\epsilon$ in Algorithm 1. 


\section{Acknowledgments}

This project is supported by the Natural Science Foundation of China (Grants nos. 11401438 and 11571120), Shandong Provincial Natural Science Foundation (Grant no. ZR2017LA002), and the Project of Shandong Province Higher Educational Science and Technology Program (Grant no. J14LI52).

\section{References}

[1] Y. Censor and T. Elfving, "A multiprojection algorithm using Bregman projections in a product space," Numerical Algorithms, vol. 8, no. 2-4, pp. 221-239, 1994.

[2] Y. Censor, T. Bortfeld, N. Martin, and A. Trofimov, "A unified approach for inversion problems in intensity-modulated radiation therapy," Physics in Medicine and Biology, vol. 51, no. 10, pp. 2353-2365, 2006.

[3] Y. Censor, A. Motova, and A. Segal, "Perturbed projections and subgradient projections for the multiple-sets split feasibility problem," Journal of Mathematical Analysis and Applications, vol. 327, no. 2, pp. 1244-1256, 2007.

[4] B. Qu and N. Xiu, "A new halfspace-relaxation projection method for the split feasibility problem," Linear Algebra and its Applications, vol. 428, no. 5-6, pp. 1218-1229, 2008.

[5] B. Qu, B. Liu, and N. Zheng, "On the computation of the stepsize for the CQ-like algorithms for the split feasibility problem," Applied Mathematics and Computation, vol. 262, pp. 218-223, 2015.

[6] Q. Yang, "The relaxed CQ algorithm solving the split feasibility problem," Inverse Problems, vol. 20, no. 4, pp. 1261-1266, 2004.

[7] H. Zhang and Y. Wang, "A new CQ method for solving split feasibility problem," Frontiers of Mathematics in China, vol. 5, no. 1, pp. 37-46, 2010.

[8] A. Moudafi, "A relaxed alternating CQ-algorithm for convex feasibility problems," Nonlinear Analysis: Theory, Methods \& Applications, vol. 79, pp. 117-121, 2013.

[9] H. Xu, "Iterative methods for the split feasibility problem in infinite-dimensional Hilbert spaces," Inverse Problems, vol. 26, 17 pages, 2010.

[10] Y. Yao, M. Postolache, and Y. Liou, "Strong convergence of a selfadaptive method for the split feasibility problem," Fixed Point Theory and Applications, vol. 2013, article 201, 2013.

[11] Y. Censor, T. Elfving, N. Kopf, and T. Bortfeld, "The multiplesets split feasibility problem and its applications for inverse problems," Inverse Problems, vol. 21, no. 6, pp. 2071-2084, 2005.

[12] B. Liu, B. Qu, and N. Zheng, "A successive projection algorithm for solving the multiple-sets split feasibility problem," Numerical Functional Analysis and Optimization, vol. 35, no. 11, pp. 1459-1466, 2014.

[13] B. Qu and H. Chang, "Remark on the successive projection algorithm for the multiple-sets split feasibility problem," Numerical Functional Analysis and Optimization, vol. 38, no. 12, pp. 1614-1623, 2017.

[14] X. Wang, "Alternating proximal penalization algorithm for the modified multiple-sets split feasibility problems," Journal of Inequalities and Applications, vol. 48, pp. 1-8, 2018.

[15] Y. Censor and A. Segal, "The split common fixed point problem for directed operators," Journal of Convex Analysis, vol. 16, no. 2, pp. 587-600, 2009.
[16] A. Moudafi, "A note on the split common fixed-point problem for quasi-nonexpansive operators," Nonlinear Analysis: Theory, Methods \& Applications, vol. 74, no. 12, pp. 4083-4087, 2011.

[17] Y. Tang and L. Liu, "Several iterative algorithms for solving the split common fixed point problem of directed operators with applications," Optimization. A Journal of Mathematical Programming and Operations Research, vol. 65, no. 1, pp. 53-65, 2014.

[18] A. Moudafi, "Alternating CQ-algorithm for convex feasibility and split fixed-point problems," Journal of Nonlinear and Convex Analysis, vol. 15, no. 4, pp. 809-818, 2014.

[19] H. Che and M. Li, "A simultaneous iterative method for split equality problems of two finite families of strictly pseudononspreading mappings without prior knowledge of operator norms," Fixed Point Theory and Applications, vol. 2015, Article ID 1, 2015.

[20] S.-S. Chang, L. Wang, and L.-J. Qin, "Split equality fixed point problem for quasi-pseudo-contractive mappings with applications," Fixed Point Theory and Applications, vol. 2015, article 208, 2015.

[21] J. Zhao and S. Wang, "Mixed iterative algorithms for the multiple-set split equality common fixed-point problems without prior knowledge of operator norms," Optimization. A Journal of Mathematical Programming and Operations Research, vol. 65 , no. 5, pp. 1069-1083, 2016.

[22] M. Li, X. Kao, and H. Che, "A simultaneous iteration algorithm for solving extended split equality fixed point problem," Mathematical Problems in Engineering, vol. 2017, Article ID 9737062, 9 pages, 2017.

[23] S. Chang, "Some problems and results in the study of nonlinear analysis," Nonlinear Analysis: Theory, Methods \& Applications, vol. 30, no. 7, pp. 4197-4208, 1997.

[24] B. T. Polyak, "Minimization of unsmooth functionals," USSR Computational Mathematics and Mathematical Physics, vol. 9, no. 3, pp. 14-29, 1969.

[25] M. Fukushima, "A relaxed projection method for variational inequalities," Mathematical Programming, vol. 35, no. 1, pp. 5870, 1986.

[26] R. Rockafellar, Convex Analysis, Princeton University Press, Princeton, NJ, USA, 1970. 


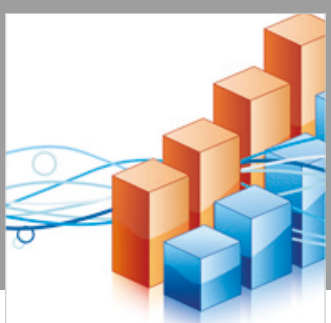

Advances in

Operations Research

\section{-n-m}
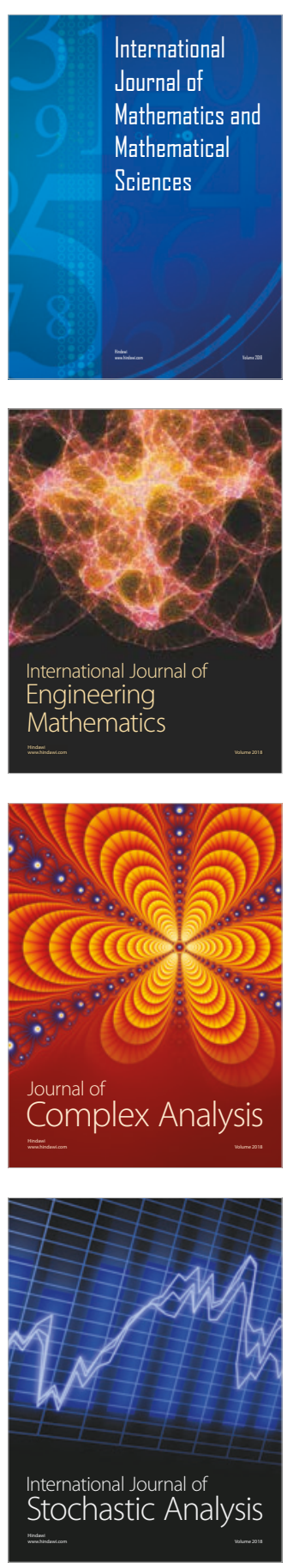
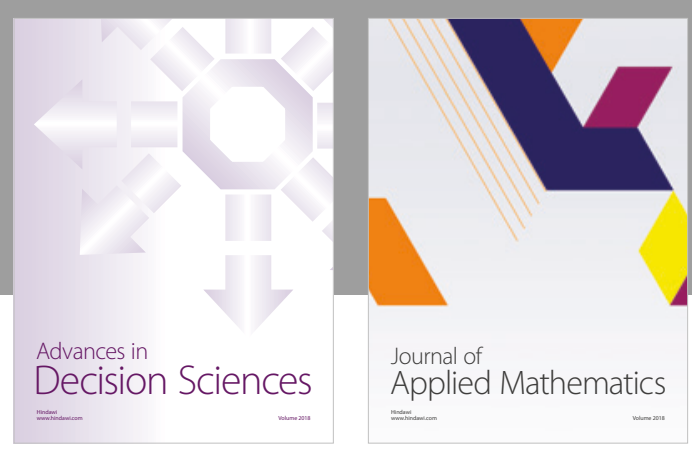

Journal of

Applied Mathematics
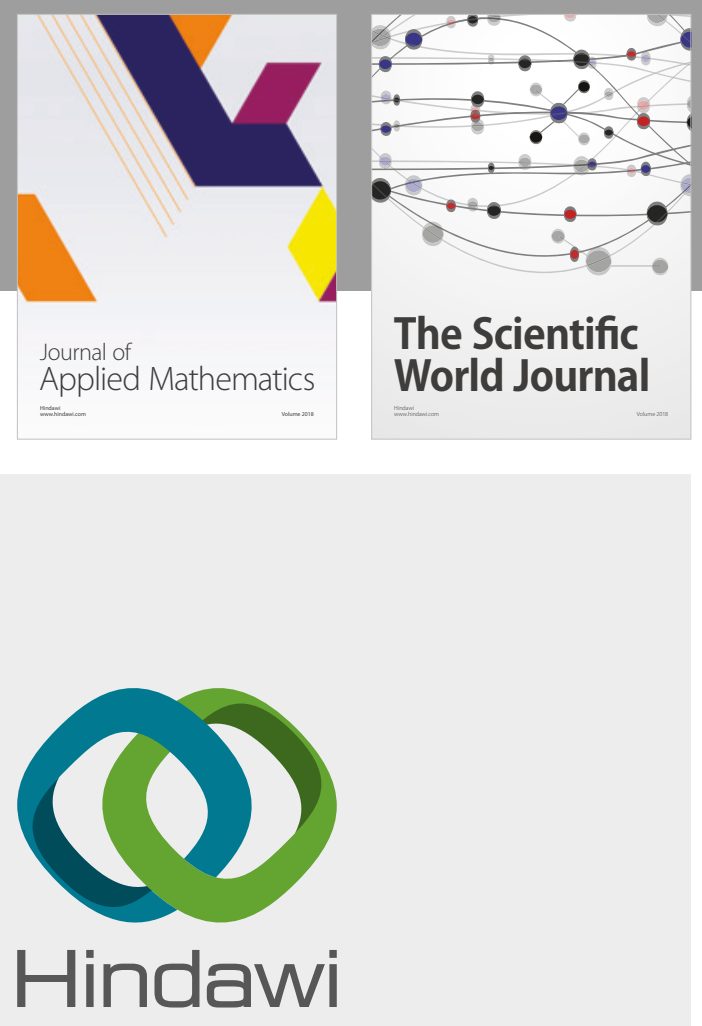

Submit your manuscripts at

www.hindawi.com

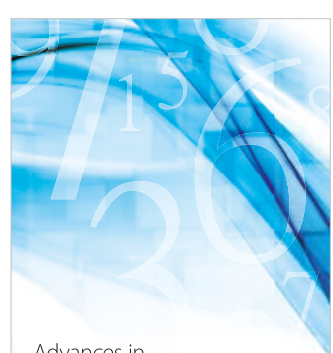

Advances in
Numerical Analysis
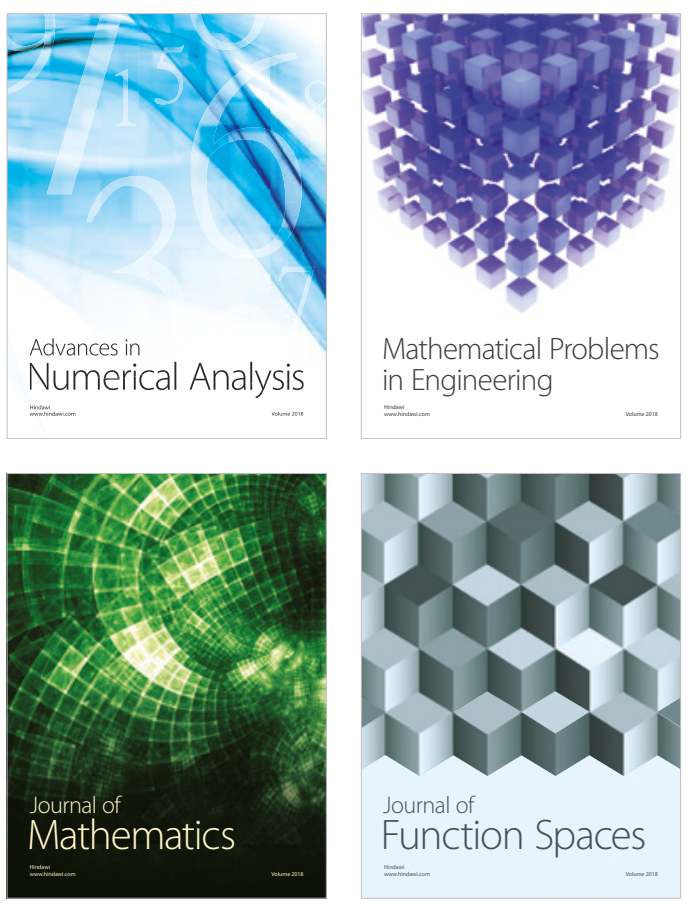

Mathematical Problems in Engineering

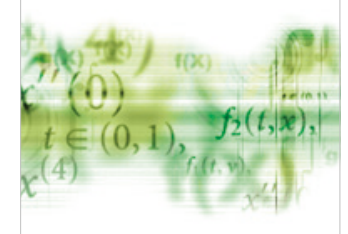

International Journal of

Differential Equations

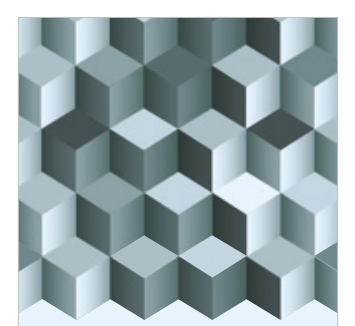

Journal of

Function Spaces

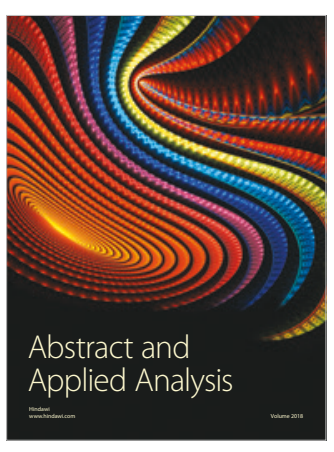

The Scientific

World Journal

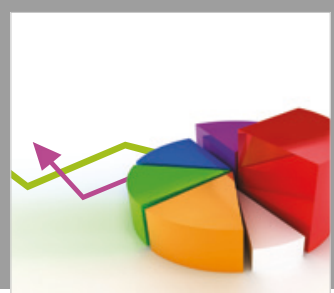

Journal of

Probability and Statistics
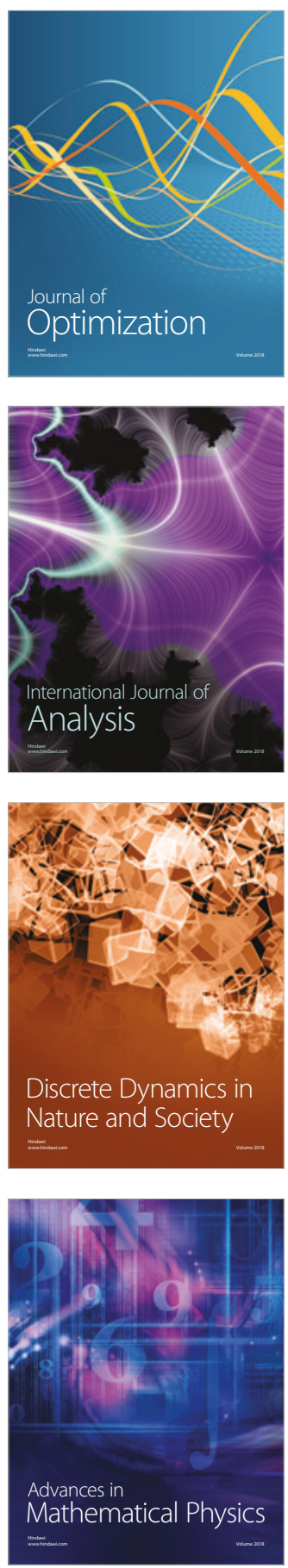